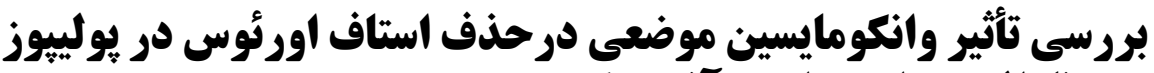

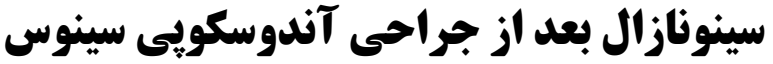

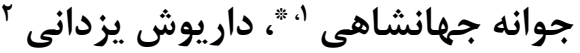

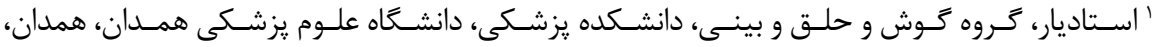

ايران

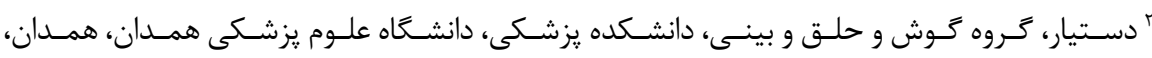

ايران

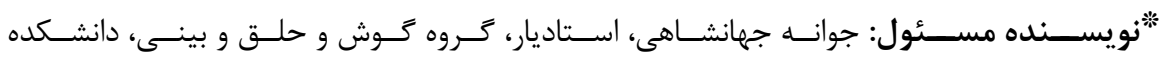

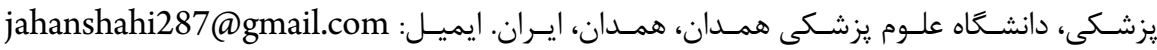

DOI: $10.21859 /$ hums-23041

مقكديده

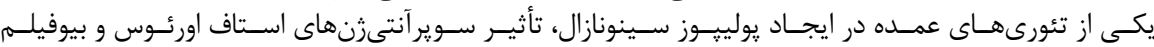

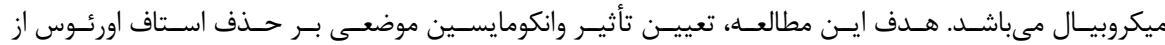

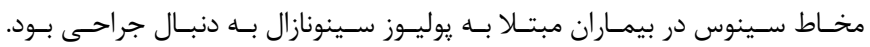

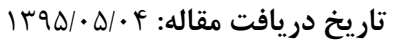
تاريخ يذيرش مقاله:

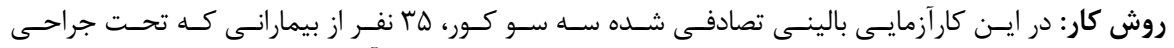

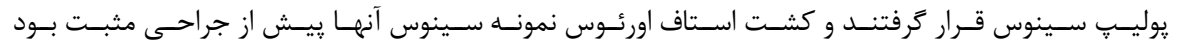

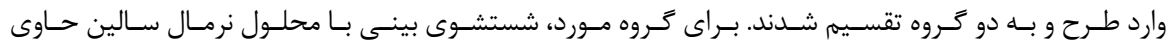

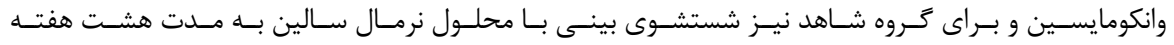

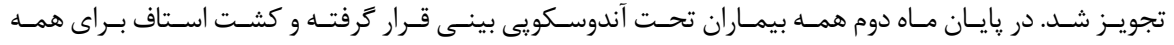

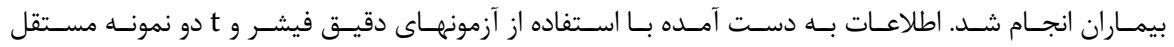

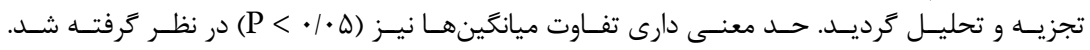

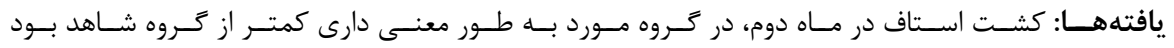
. $(\mathrm{P}<\cdot / \cdot \Delta)$

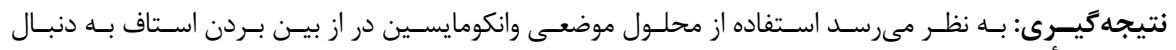

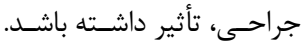

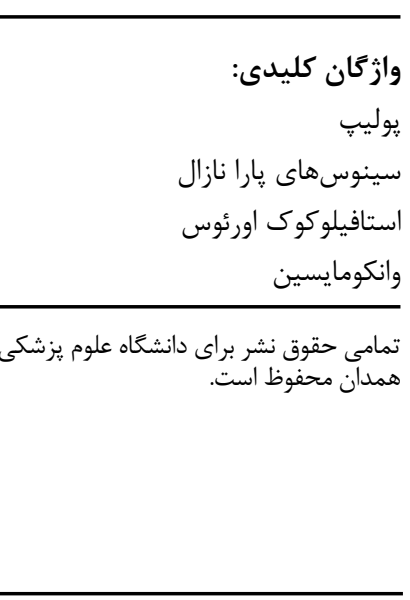

وازًَان كليدى:

يوليب

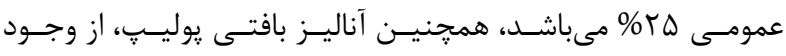

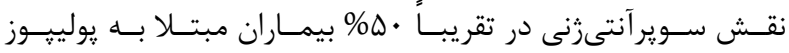

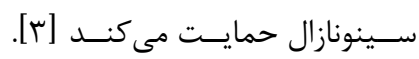

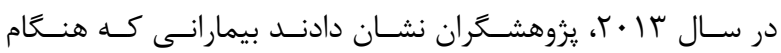

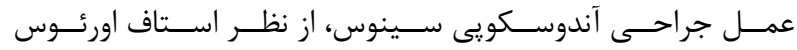

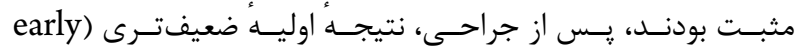
postoperative outcomes

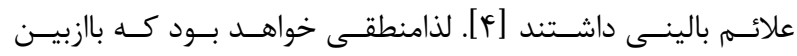

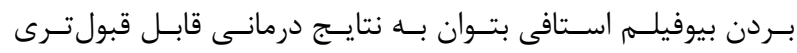

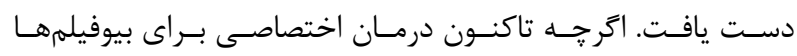

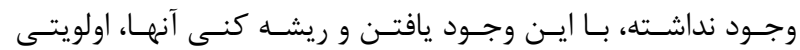

مهـهم بـه شـمار مسىرود.

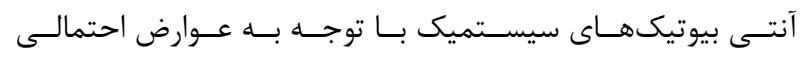

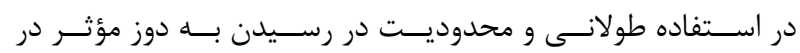

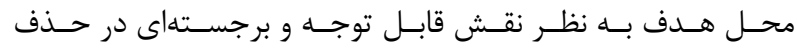

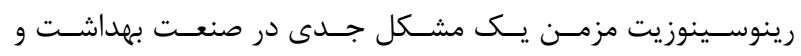

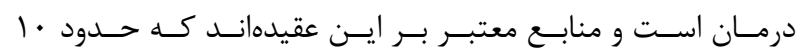

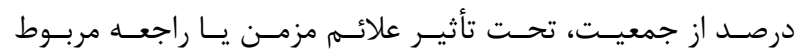

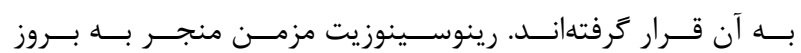

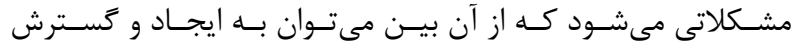

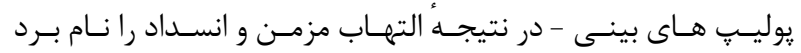

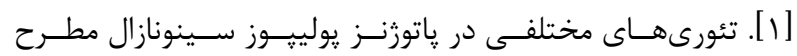

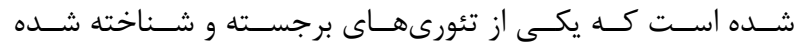

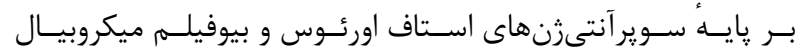
مىباشــد [ب]. اســافيلوكوك اورئسوس شـايعترين باكتـــى جــــا شــده از موكـوس

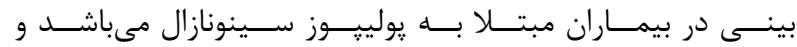

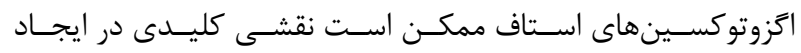

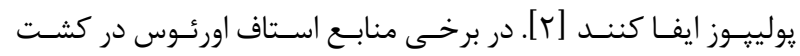

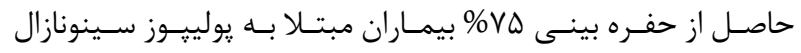




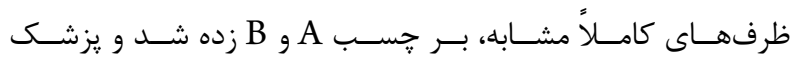

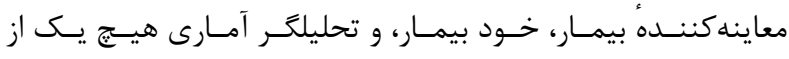

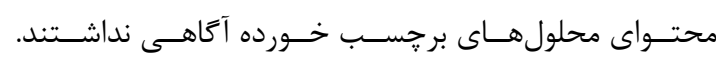

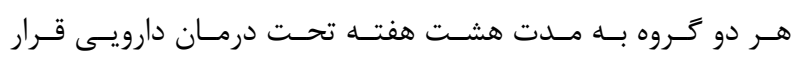
كرفتنـد:

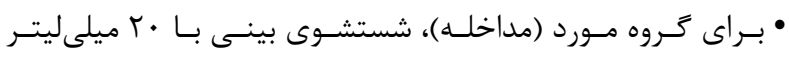

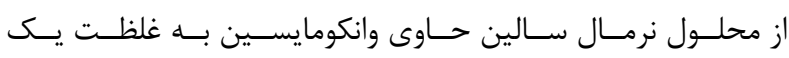

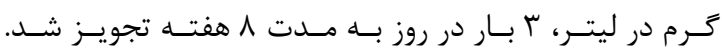

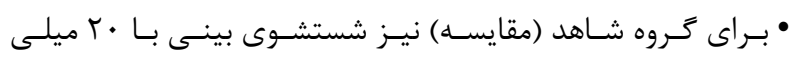

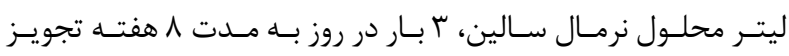
شد .

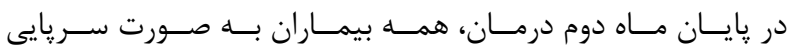

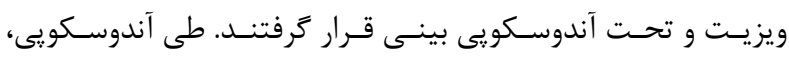

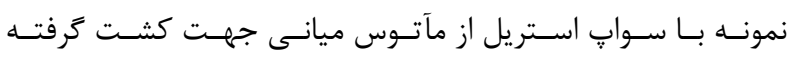

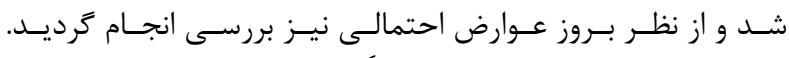

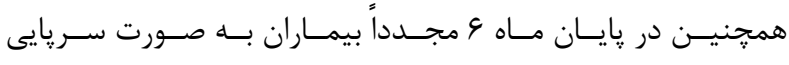

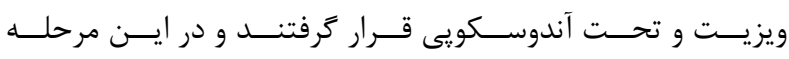

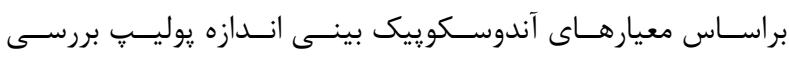

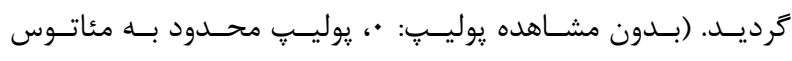

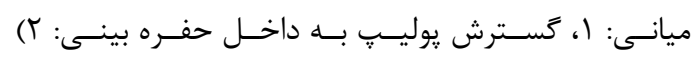

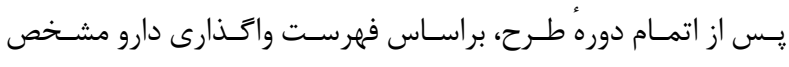

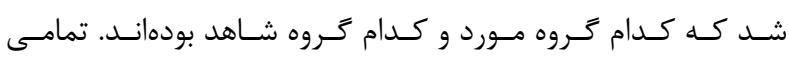

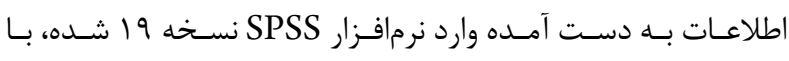

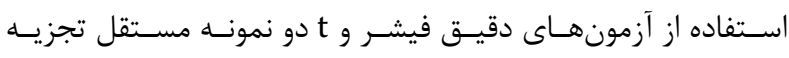

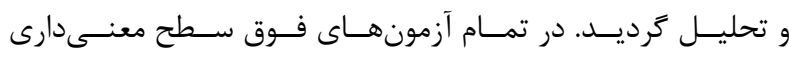

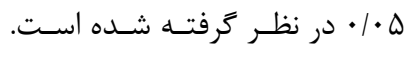

\section{يافتهها}

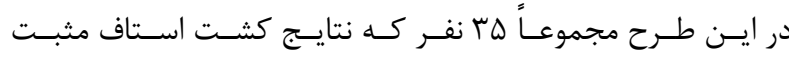

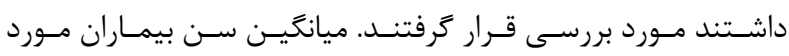

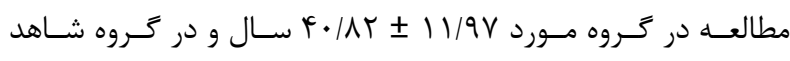

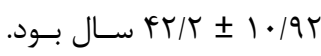

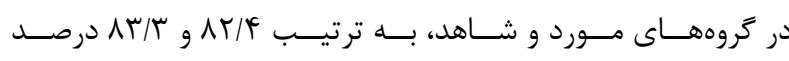

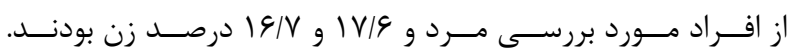

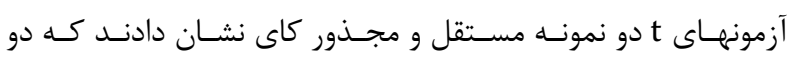

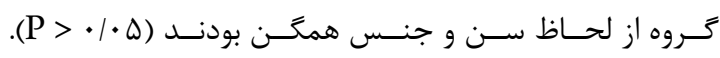

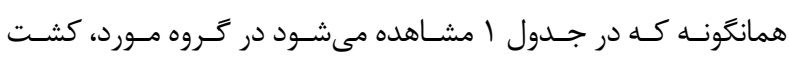

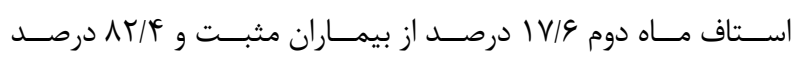

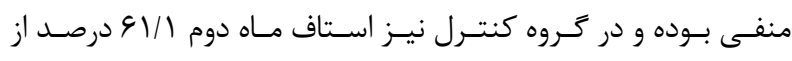

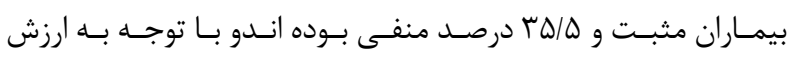

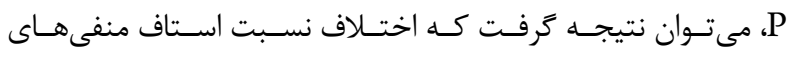

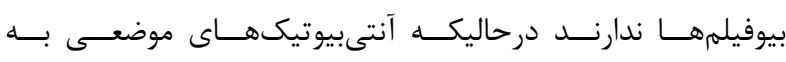

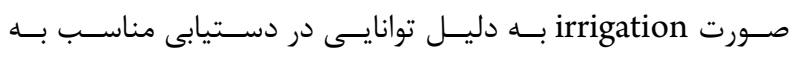

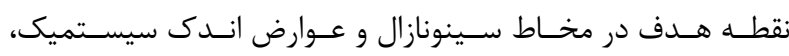

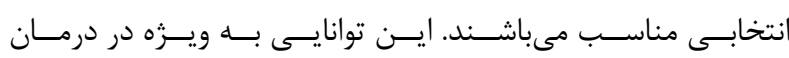

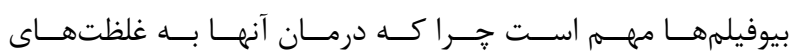

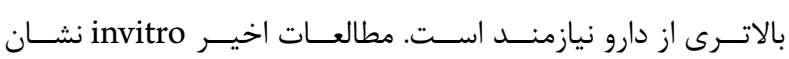

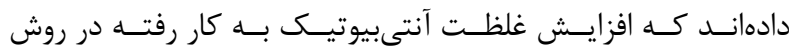

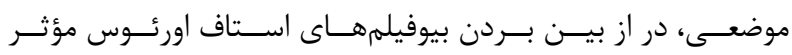
اسـت [هـ] موضع.

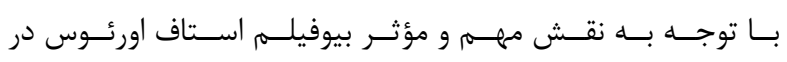

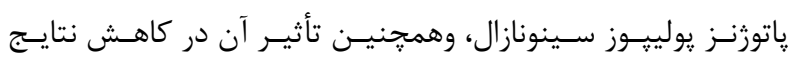

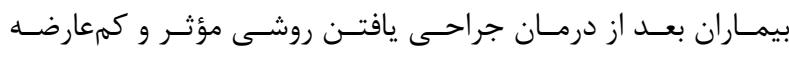

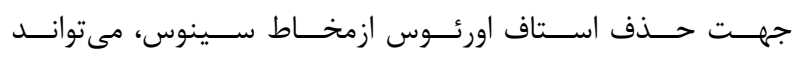

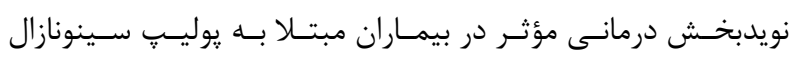

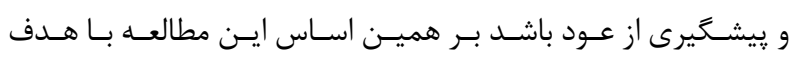

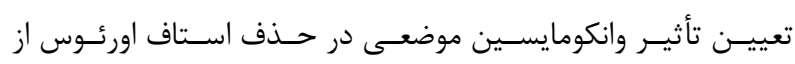

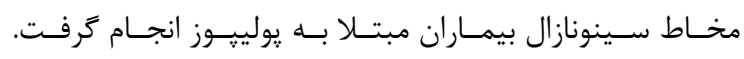

\section{روش كار}

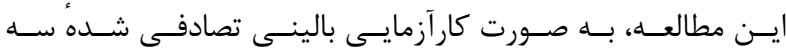

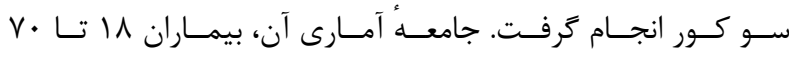

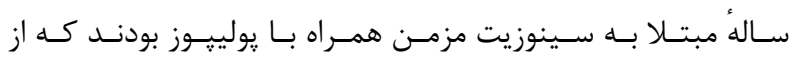

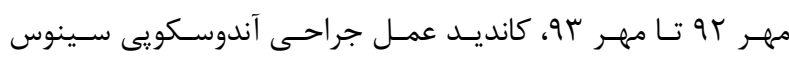

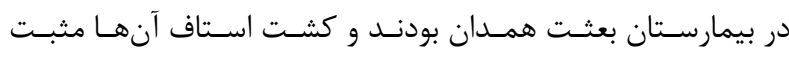

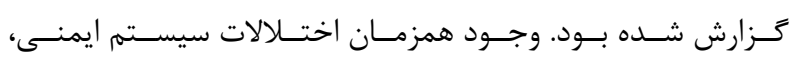

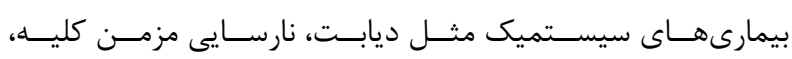

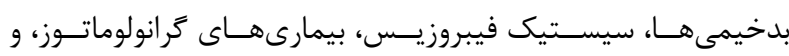

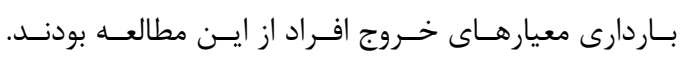

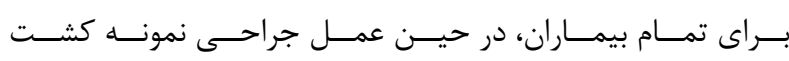

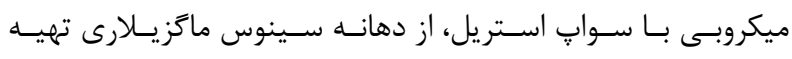

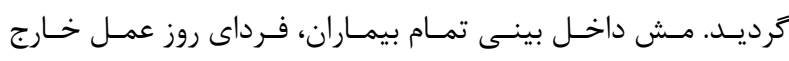

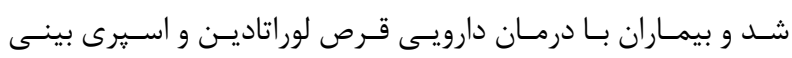

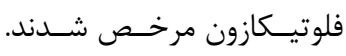

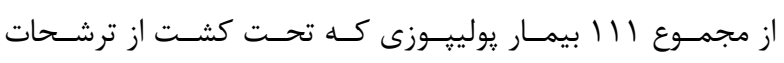

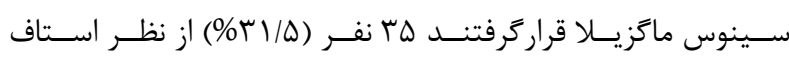

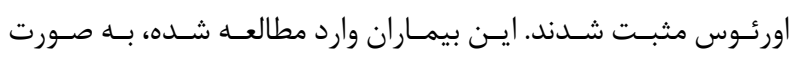

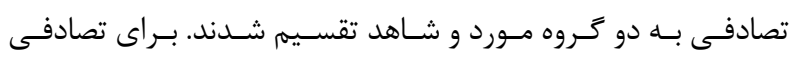
سـازى از روش بركههـــاى تصادفـى (block randomization)

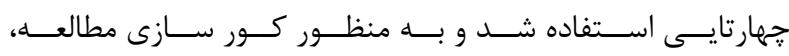
تهيـهـ محلولهـاى حــاوى وانكومايسـين (مداخلـه) و نرمـال سـالين

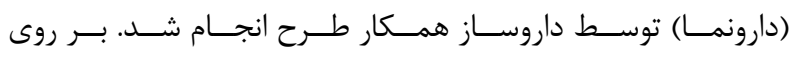




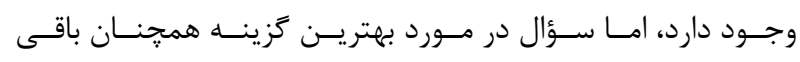

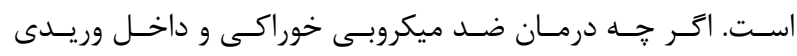

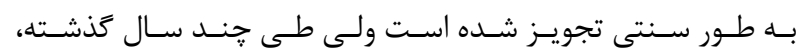

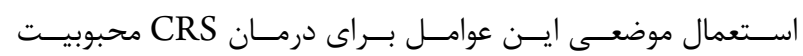

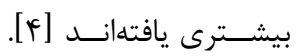

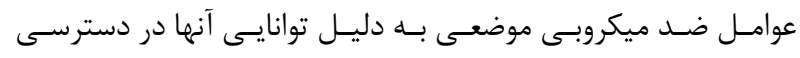

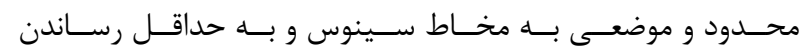

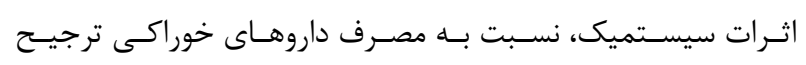

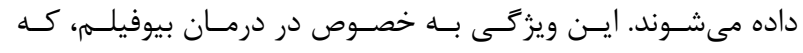

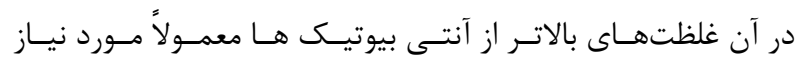

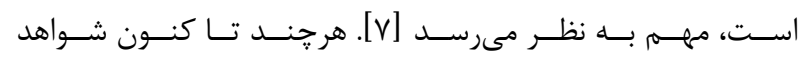

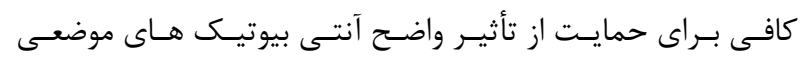

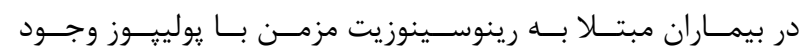

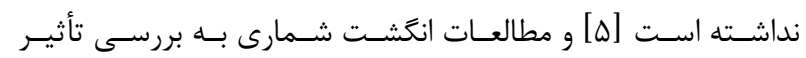

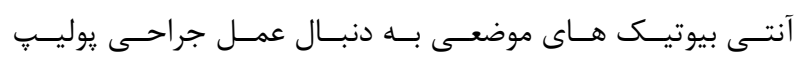

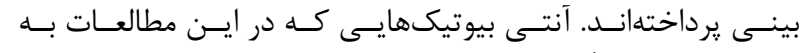

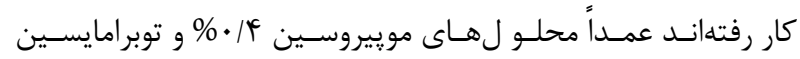

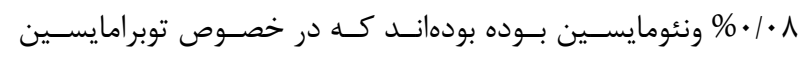

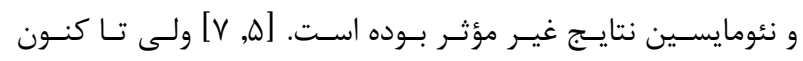

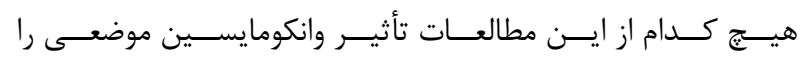

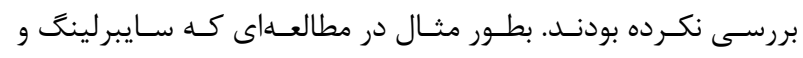

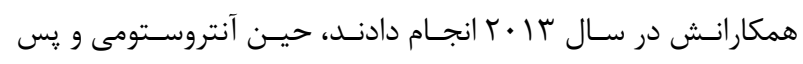

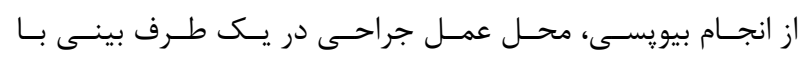

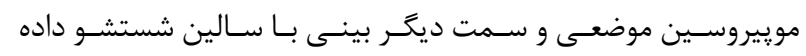

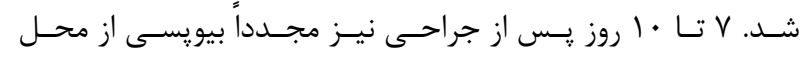

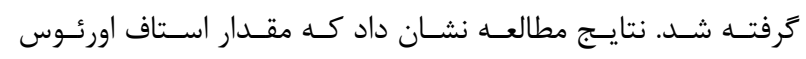

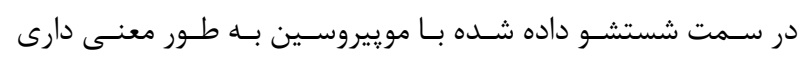

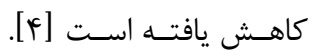

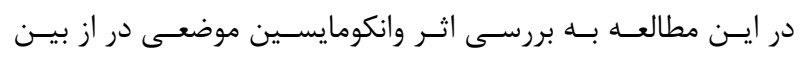

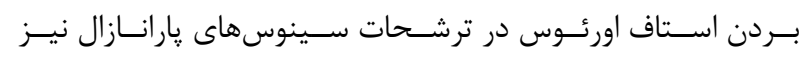

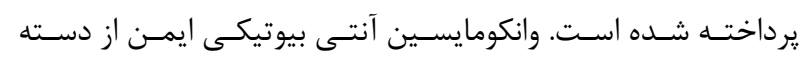

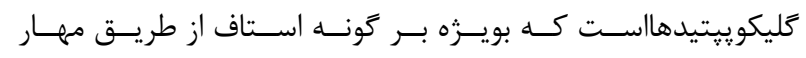

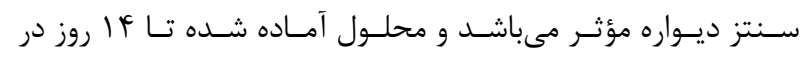

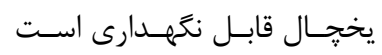

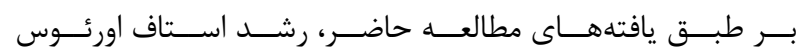

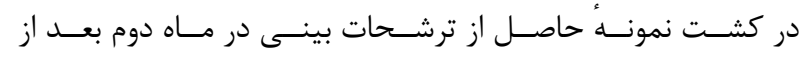

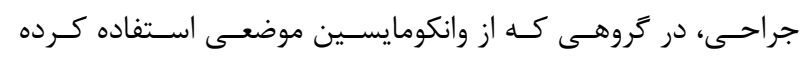

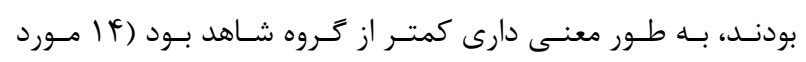

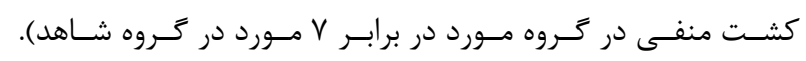

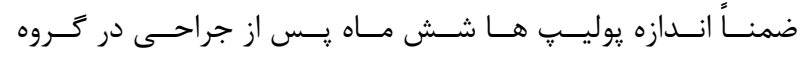

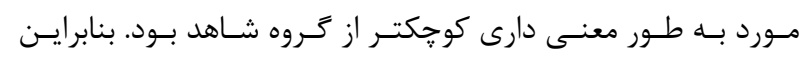

مـاه دوم در دو گَـروه آزمسون و كنتـرل معنسى دار بـوده اسـت.

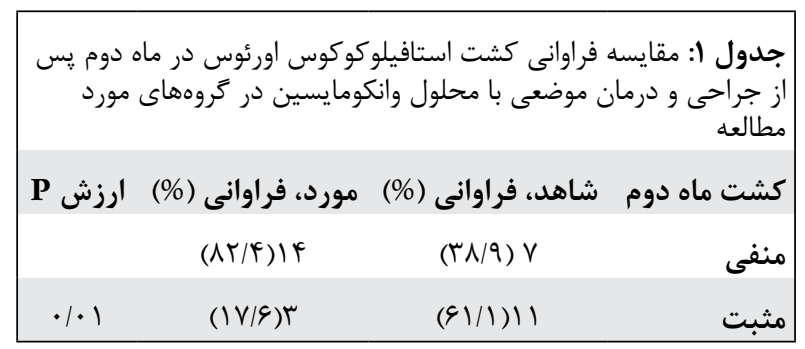

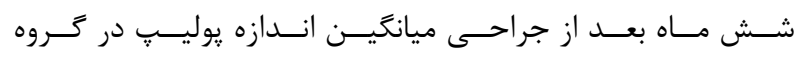

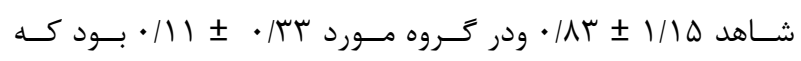

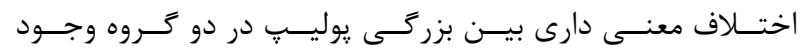

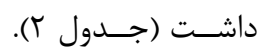

\begin{tabular}{|c|c|c|c|}
\hline \multicolumn{4}{|c|}{ ماه يسول بـ مقايسهُ تأثير مداخله انجام شده بر اندازه يوليٍ در قبل و شش } \\
\hline ارزش P & مورد، فراوانى (\%) & شاهد، فراوانى (\%) & بزركى يولي" \\
\hline .1 .9 & $(\cdot / V F) r / \cdot \Delta$ & $(\cdot / A \vee) \Psi / / \varepsilon$ & قبل از جراحى \\
\hline $.1 \cdot 1$ & ( $) \cdot / 11$ & $(1 / / \Delta) \cdot / \wedge r$ & از شر ماه بعد \\
\hline
\end{tabular}

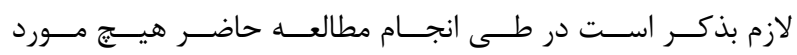

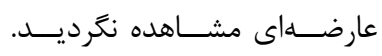

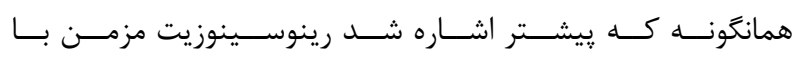

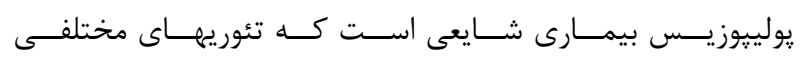

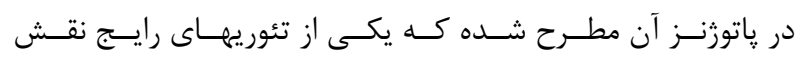

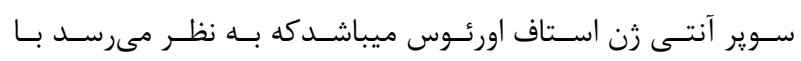

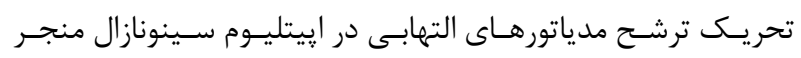

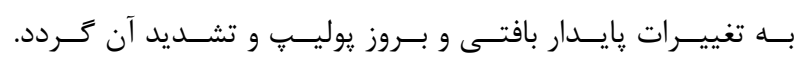

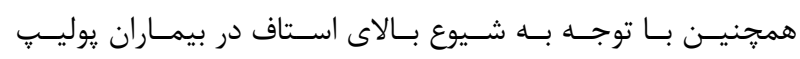

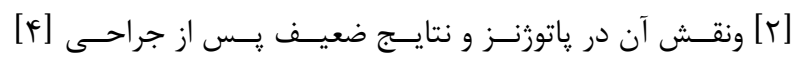

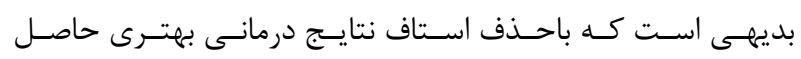
شـود.

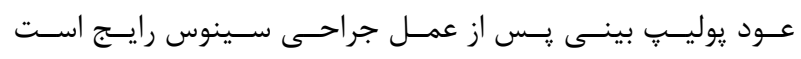

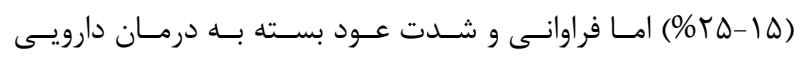

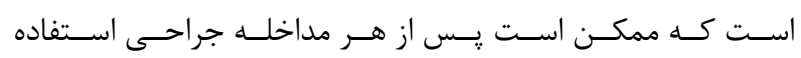

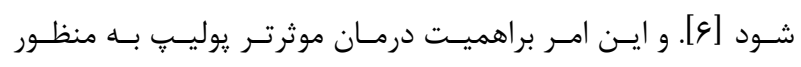
ييشـــيرى از عــود مى افزايــد.

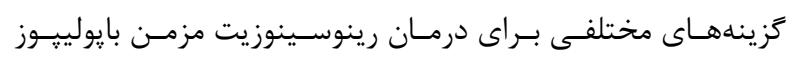


عارضـهـ حــذف اسـتاف بتـوان شـاهد كاهـش عـود يوليسِ بعـد از

$$
\text { جراحسى بـود. }
$$

سياسگز إوى

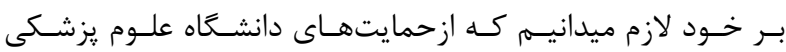

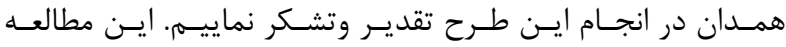

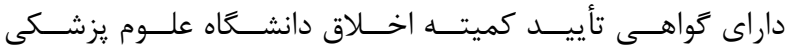

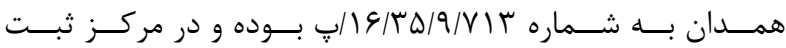

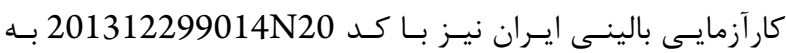

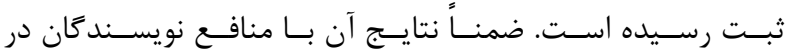
تعـارض نيست.

\section{REFERENCES}

1. Flint P, Haughey B, Lund V, Niparko J, Robbins T, Thomas R. Cummings otolaryngology: head and neck surgery. 6th ed. Philadelphia: Saunders; 2014.

2. Bernstein JM, Allen C, Rich G, Dryja D, Bina P, Reiser R, et al Further observations on the role of Staphylococcus aureus exotoxins and $\operatorname{IgE}$ in the pathogenesis of nasal polyposis. Laryngoscope. 2011;121(3):647-55. DOI: 10.1002/lary.21400 PMID: 21344448

3. Karosi T, Sziklai I, Csomor P. Low-frequency ultrasound for biofilm disruption in chronic rhinosinusitis with nasal polyposis: in vitro pilot study. Laryngoscope. 2013;123(1):17-23. DOI: 10.1002/lary.23633 PMID: 22893599

4. Seiberling KA, Aruni W, Kim S, Scapa VI, Fletcher H, Church
مى تـوان نتيجـهـ كرفــت كـهـ مداخلـهُ مـا (اسـتفاده از وانكومايسـين

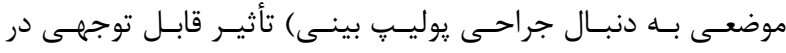

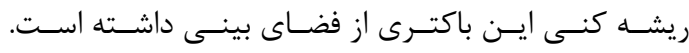

\section{نتيجه كيرى}

بـا در نظــر كرفتـن همــه مـوارد فـوق، بــه نظــر مىرســ اسـتفاده

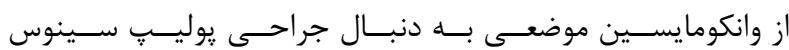

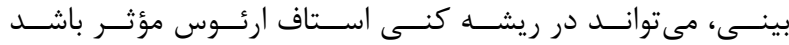

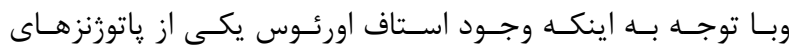

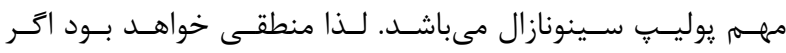

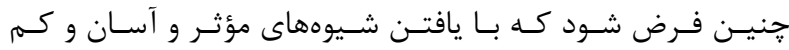

CA. The effect of intraoperative mupirocin irrigation on Staphylococcus aureus within the maxillary sinus. Int Forum Allergy Rhinol. 2013;3(2):94-8. DOI: 10.1002/alr.21076 PMID: 23192968

5. Wei CC, Adappa ND, Cohen NA. Use of topical nasal therapies in the management of chronic rhinosinusitis. Laryngoscope. 2013;123(10):2347-59. DOI: 10.1002/lary.24066 PMID: 23553558

6. Esmatinia F. Recurrent sinonasal polyposis after the endoscopic sinus surgery. Rev Clin Med. 2014;1(2):86-92.

7. Comstock RH, 3rd, Lam K, Mikula S. Topical antibiotic therapy of chronic rhinosinusitis. Curr Infect Dis Rep. 2010;12(2):88-95. DOI: 10.1007/s11908-010-0085-4 PMID: 21308504 


\title{
Effect of Topical Vancomycin on Eradication of Staphylococcus aureus in Sinonasal Polyposis After Functional Endoscopic Sinus Surgery
}

\author{
Javaneh Jahanshahi ${ }^{1,}{ }^{*}$, Darush Yazdani ${ }^{2}$ \\ ${ }^{1}$ Assistant Professor, Department of ENT, Faculty of Medicine, Hamadan University \\ of Medical Sciences, Hamadan, Iran \\ ${ }^{2}$ Resident, Department of ENT, Faculty of Medicine, Hamadan University of Medical \\ Sciences, Hamadan, Iran \\ ${ }^{*}$ Corresponding author:Javaneh Jahanshahi, Assistant Professor, Department of \\ ENT, Faculty of Medicine, Hamadan University of Medical Sciences, Hamadan, \\ Iran.E-mail: jahanshahi287@gmail.com
}

DOI: $10.21859 /$ hums-23041

Received: 25.07.2016

Accepted: 18.12.2016

Keywords:

Polyps

Nasal Sinuses

Staphylococcus aureus

Vancomycin

How to Cite this Article:

Jahanshahi J, Yazdani D. Effect of Topical Vancomycin on Eradication of Staphylococcus aureus in Sinonasal Polyposis After Functional Endoscopic Sinus Surgery. Sci J Hamadan Univ Med Sci. 2017;23(4):288-292. DOI: 10.21859 /hums-23041

(C) 2017 Hamadan University of Medical Sciences.

\section{Abstract}

Introduction: One of the main theories in development of sinonasal polyposis involves the effect of Staphylococcus aureus super antigens and microbial biofilm. The aim of this study was to evaluate the effect of topical vancomycin on Staphylococcus aureus removal from sinus mucosa following surgery.

Methods: In this randomized triple-blind clinical trial, 35 patients, who underwent sinus polyp surgery and whose sinus culture was positive for Staphylococcus aureus before surgery, were divided to two groups. For the case group, nasal irrigation with normal saline solution containing vancomycin and for the control group, nasal irrigation with normal saline solution was administered for eight weeks. During the second month of treatment, all patients underwent nasal endoscopy. Patients were assessed for Staphylococcus aureus culture during the second month. All data were analyzed using two independent samples t-test and Fisher's exact tests. The level of statistical significance was considered as $\mathrm{P}<0.05$. Results: Culture of Staphylococcus aureus in the second month was significantly lower in the case group than the control one $(\mathrm{P}<0.05)$.

Conclusion: It seems that the use of topical vancomycin solution has an effect on eradication of Staphylococcus aureus in sinonasal polyposis after surgery. 